\title{
Generating Function Approach to the Derivation of Higher-Order Iterative Methods for Solving Nonlinear Equations
}

\author{
Tugal Zhanlav ${ }^{1, \star}$, Ochbadrakh Chuluunbaatar ${ }^{1,2, \star \star}$, and Vandandoo Ulziibayar ${ }^{1,3, \star \star \star}$ \\ ${ }^{1}$ Institute of Mathematics, National University of Mongolia, Mongolia \\ ${ }^{2}$ Joint Institute for Nuclear Research, Dubna, 141980 Moscow region, Russia \\ ${ }^{3}$ School of Applied Sciences, Mongolian University of Science and Technology, Mongolia
}

\begin{abstract}
In this paper we propose a generating function method for constructing new two and three-point iterations with $p(p=4,8)$ order of convergence. This approach allows us to derive a new family of optimal order iterative methods that include well known methods as special cases. Necessary and sufficient conditions for $p$-th $(p=4,8)$ order convergence of the proposed iterations are given in terms of parameters $\tau_{n}$ and $\alpha_{n}$. We also propose some generating functions for $\tau_{n}$ and $\alpha_{n}$. We develop a unified representation of all optimal eighth-order methods. The order of convergence of the proposed methods is confirmed by numerical experiments.
\end{abstract}

\section{Introduction}

Solving nonlinear equations is important in many applied mathematics and theoretical physics problems. In recent years, a number of higher-order iterative methods have been developed and analyzed on this issue, see [1-11] and references therein. Motivated by the recent results in [11], in this paper we introduce a generating function method for the construction of new two and three-point iterations with $p$-th order of convergence. This paper is organized as follows. Section 2 is devoted to the construction of a generating function for the optimal fourth-order method. We then present some choices for the parameters $\tau_{n}$ and $\alpha_{n}$. Some iterations are proposed among which some are already well known. In Section 3 we propose a family of optimal eighth-order methods, that include many well-known methods as particular cases. In our previous paper [11] we have considered two and three-point iterative methods of solving nonlinear equation $f(x)=0$

$$
y_{n}=x_{n}-\frac{f\left(x_{n}\right)}{f^{\prime}\left(x_{n}\right)}, \quad x_{n+1}=y_{n}-\bar{\tau}_{n} \frac{f\left(y_{n}\right)}{f^{\prime}\left(x_{n}\right)},
$$

and

$$
y_{n}=x_{n}-\frac{f\left(x_{n}\right)}{f^{\prime}\left(x_{n}\right)}, \quad z_{n}=y_{n}-\bar{\tau}_{n} \frac{f\left(y_{n}\right)}{f^{\prime}\left(x_{n}\right)}, \quad x_{n+1}=z_{n}-\alpha_{n} \frac{f\left(z_{n}\right)}{f^{\prime}\left(x_{n}\right)} .
$$

We have proved in [11] the following theorems:

\footnotetext{
^e-mail: tzhanlav@yahoo.com

$\star \star$ e-mail: chuka@jinr.ru

$\star \star \star$ e-mail: ulzii@jinr.ru
} 
Theorem 1. Assume that $f(x)$ is smooth enough function with a simple root $x^{*} \in I$ and the initial approximation $x_{0}$ is close enough to $x^{*}$. Then the iterative method (1) has fourth-order of convergence if and only if the parameter $\tau_{n}$ is given by

$$
\tau_{n}=1+\theta_{n}+2 \theta_{n}^{2}+O\left(\theta_{n}^{3}\right), \quad \theta_{n}=\frac{f\left(y_{n}\right)}{f\left(x_{n}\right)} .
$$

Theorem 2. Assume that all assumptions of Theorem 1 are fulfilled. Then the three-point iterative methods (2) has an eighth-order of convergence if and only if the parameters $\bar{\tau}_{n}$ and $\alpha_{n}$ are given by

$$
\bar{\tau}_{n}=1+2 \theta_{n}+\beta \theta_{n}^{2}+\gamma \theta_{n}^{3}+\cdots, \quad\left(\bar{\tau}_{n}=\frac{\tau_{n}-1}{\theta_{n}}\right),
$$

and

$$
\alpha_{n}=1+2 \theta_{n}+(\beta+1) \theta_{n}^{2}+(2 \beta+\gamma-4) \theta_{n}^{3}+\left(1+4 \theta_{n}\right) \frac{f\left(z_{n}\right)}{f\left(y_{n}\right)}+O\left(\theta_{n}^{4}\right)
$$

Our approach in [11] is constructive in the sense that it proposes a new way to obtain optimal order iterations (see [11] for details). An extended version of the present paper will be published elsewhere.

\section{Construction of optimal fourth-order methods}

The Theorems 1 and 2 not only give sufficient conditions for iterations of $p$-th $(p=4,8)$ order of convergence, but they also allow us to construct new iterations with $p$ order of convergence. Obtaining new optimal methods of order four is still important, because they combine higher-order of convergence and low computational cost. We consider the following choice of the parameter $\tau_{n}$

$$
\tau_{n}=H\left(\theta_{n}\right),
$$

where $H(\theta)$ is a real function to be determined properly. Obviously $\tau_{n}$ will satisfy the condition (3) if

$$
H(0)=1, \quad H^{\prime}(0)=1, \quad H^{\prime \prime}(0)=4 .
$$

We call the function $H(\theta)$ satisfying conditions (7) a generating function for the iteration (1). The construction of the generating function allows us to derive a new optimal order family of iterations. The following theorem is a consequence of Theorem 1.

Theorem 3. Assume that all assumptions of Theorem 1 are fulfilled. Then the optimal fourth-order two-point iterations (1) are obtained by the generating function (6) satisfying the conditions (7).

Many different variants of the generating function $H(x)$, satisfying condition (7) are possible. We cite here one simple form, namely

$$
H(x)=\frac{1+(1-m \alpha) x+\left(2-m \alpha+\frac{m(m-1)}{2} \alpha^{2}\right) x^{2}+\omega x^{3}}{(1-\alpha x)^{m}}, \quad \alpha, m, \omega \in \mathbb{R} .
$$

The optimal two-point iterations (1) with $\tau_{n}=H\left(\theta_{n}\right)$ given by (8) include many well-known iterations as special cases. If $\omega=0, m=1$ and $\alpha=2-b, b \in \mathbb{R}$, then (1) leads to King's method [5]. If $\alpha=0, m=1$ and $\omega=1$ in (8), then (1) yields a modification of Potra-Ptak's method [4]. If $\alpha=m=1$ and $\omega=-1$ in (8), then (1) leads to Maheshwari's method [7]. If $\alpha=1, m=2$ and $\omega=0$ in (8), then (1) leads to Chun and Lee's method [2]. Recently, Behl et al [12] proposed a general class of fourthorder optimal methods that includes the well-known Ostrowski's and King's family as special cases. We note that this general class of optimal fourth-order iterations is also included in our methods with 
$\tau_{n}=H\left(\theta_{n}\right)$ given by (8) as a special case. Namely, if $m=3, \alpha$ replaced by $-\alpha$ and $\omega=\alpha^{2}+\frac{5}{3} \alpha+\frac{4}{3}$ or $\omega=\left(1-\frac{\beta}{6}\right) \alpha^{3}+\alpha^{2}-2 \alpha$, then the iterations (1) with $\tau_{n}=H\left(\theta_{n}\right)$ given by (8) reduce to (3.8) and (3.10) in [12], respectively. This shows that our class of optimal fourth-order methods is wider than that of [12]. So, we have obtained an optimal fourth-order convergence family of iterative methods with three degrees of freedom based on the generating function method.

\section{Proper representation of the optimal order three-point iterative methods}

Recently, based on optimal fourth-order methods some higher-order, in particular eighth order threepoint methods have been proposed for solving nonlinear equations. It is easy to show that $\tau_{n}=H\left(\theta_{n}\right)$ given by (8) satisfies the condition (4) provided

$$
\begin{aligned}
& \beta=\omega+2 m \alpha-\frac{m(m-1)}{2} \alpha^{2}+\frac{m(m-1)(m-2)}{6} \alpha^{3}, \\
& \gamma=\omega m \alpha+m(m+1) \alpha^{2}-\frac{(m-1) m(m+1)}{3} \alpha^{3}+\frac{(m-2)(m-1) m(m+1)}{8} \alpha^{4} .
\end{aligned}
$$

Table 1. Optimal order three-point iterative methods recovered at special parameter values

\begin{tabular}{|c|c|c|l|}
\hline & $m$ & $\alpha_{n}-\left(1+4 \theta_{n}\right)\left[f\left(z_{n}\right) / f\left(y_{n}\right)\right]$ & Methods \\
\hline 1 & 0 & $1+2 \theta_{n}+(\beta+1) \theta_{n}^{2}+(2 \beta+\gamma-4) \theta_{n}^{3}$ & $\begin{array}{l}\beta=\gamma=1,[14] \text { Maheshwari-based } \\
\beta=4, \gamma=8, \text { Method 1 in [14] see [11] } \\
\beta=3, \gamma=4, \text { Chun Lee [2] }\end{array}$ \\
\hline 2 & 1 & $\left(2-\theta_{n}\right) /\left(6 \theta_{n}^{2}-5 \theta_{n}+2\right)$ & $\begin{array}{l}p=-3, d=0, q=5 / 2, \text { Maheshwari-based } \\
\text { optimal methods [16] }\end{array}$ \\
\hline 3 & 1 & {$\left[2 \beta-1+2\left(\beta^{2}-4 \beta+1\right) \theta_{n}+(1+4 \beta) \theta_{n}^{2}\right]$} & $\begin{array}{l}d=0, q=2\left(\beta^{2}-4 \beta+1\right) /(1-2 \beta), \\
p=(1+4 \beta) /(1-2 \beta), \text { King-based optimal methods [17] }\end{array}$ \\
\hline 4 & 1 & $1 /\left(1-2 \theta_{n}-\theta_{n}^{2}\right)$ & $\begin{array}{l}\beta=4, \gamma=8, q=2, p=1, d=\omega=0, \\
\text { method 4in [14] }\end{array}$ \\
\hline
\end{tabular}

The following theorem is a consequence of Theorem 2.

Theorem 4. Assume that all assumptions of Theorem 1 are fulfilled. Then the family of three-point iterative methods (2) has an eighth-order of convergence if and only if the parameters $\tau_{n}$ and $\alpha_{n}$ are given by (6), (8) and

$$
\alpha_{n}=\left(H\left(\theta_{n}\right)+\theta_{n}+(\beta-1) \theta_{n}^{2}+(\beta+\gamma-4) \theta_{n}^{3}\right)+\left(1+4 \theta_{n}\right) \frac{f\left(z_{n}\right)}{f\left(y_{n}\right)} .
$$

Thus, we propose the families of three-point iterative methods (2) with generating function $\tau_{n}=$ $H\left(\theta_{n}\right)$. They include many well-known eighth-order methods, as particular cases (see Table 1). The expression in brackets in (10) can be approximated by a simple rational function without loss of generality. Then $\alpha_{n}$ can be represented as

$$
\alpha_{n}=\frac{1-(2-m q) \theta_{n}+c \theta_{n}^{2}+\omega \theta_{n}^{3}}{\left(1-\theta_{n}\left(d \theta_{n}^{2}+p \theta_{n}+q\right)\right)^{m}}+\left(1+4 \theta_{n}\right) \frac{f\left(z_{n}\right)}{f\left(y_{n}\right)}, \quad q, p, d, m \in \mathbb{R},
$$

where

$$
\begin{aligned}
& c=\beta+1-m\left(p+2 q+\frac{1}{2}(m-1) q^{2}\right), \\
& \omega=(2 \beta+\gamma-4)-m\left(d+2 p+(\beta+1+(1-m) p) q-(m-1) q^{2}+\frac{(m-1)(m-2)}{6} q^{3}\right) .
\end{aligned}
$$


We call the optimal order three-point iterative methods (2), with parameters $\tau_{n}$ and $\alpha_{n}$ given by (6), (8) and (11) respectively, proper representations. It is easy to show that all the well-known optimal order three-point iterative methods can be represented uniquely in the proper form (see $[1-3,6,8-$ 11, 13-20] and references therein). It should be mentioned that $\mathrm{Wu}$ and Lee in [10] first used a proper representation of (2). Thus, by means of (6), (8) and (11) we find a unified representation of all optimal order three-point iterations. It should be mentioned that the order of convergence of the proposed methods was confirmed by numerical experiments.

\section{Conclusions}

The construction of the generating function for $\tau_{n}$ and $\alpha_{n}$ allows us to derive new optimal order family of iterations. This family includes many known iterations as special cases. We develop a unified and proper representation of optimal eighth-order three-point methods. The sufficient and necessary conditions for iterations (2) to be $p(p=4,8)$ order of convergence are also given in term of parameters $\tau_{n}$ and $\alpha_{n}$.

\section{Acknowledgments}

The work was supported partially by the Foundation of Science and Technology of Mongolia under grant SST_007/2017. O. Ch. acknowledges support from the Hulubei-Meshcheryakov program JINRRomania.

\section{References}

[1] W. Bi, H.Ren, and Q. Wu, J. Comput. Appl. Math. 225, 105-112 (2009)

[2] C. Chun and M.Y. Lee, Appl. Math. Comput. 223, 506-519 (2013)

[3] A. Cordero, J.R. Torregrosa, and M.P. Vassileva, J. Comput. Appl. Math. 235, 3189-3194 (2011)

[4] A. Cordero, J.L. Hueso, E. Martinez, and J.R. Torregrosa, J. Comput. Appl. Math. 234, 29692976 (2010)

[5] R.F. King, SIAM Journal on Numerical Analysis 10, 876-879 (1973)

[6] T. Lotfi, S. Sharifi, M. Salimi, and S. Siegmund, Numer. Algor. 68, 261-288 (2015)

[7] A.K. Maheshwari, Appl. Math. Comput. 211, 383-391 (2009)

[8] S. Sarifi, M. Ferrara, M. Salimi, and S. Siegmant, Open Mathematics 14, 443-451 (2016)

[9] J.R. Sharma and R. Sharma, Numer. Algor. 54, 445-458 (2010)

[10] X. Wang and L. Liu, J. Comput. Appl. Math. 234, 1611-1620 (2010)

[11] T. Zhanlav, V. Ulziibayar, and O. Chuluunbaatar, Comput. Math. Math. Phys. 57, 1090-1100 (2017)

[12] R. Behl, A. Cordero, S.S. Motsa, and J.R. Torregrosa, Appl. Math. Comput. 271, 89-101 (2015)

[13] T. Zhanlav and V. Ulziibayar, Amer. J. Comp. Appl. Math. 6(5), 177-181 (2016)

[14] R. Thukral and M. S. Petkovic, J. Comput. Appl. Math. 233, 2278-2284 (2010)

[15] C. Chun and B. Neta, Appl. Math. Comput. 245, 86-107 (2014)

[16] C. Chun and B. Neta, Appl. Math. Comput. 253, 294-307 (2015)

[17] C. Chun and B. Neta, American Journal of Algorithms and Computing 2(1), 1-17 (2015)

[18] C. Chun and B. Neta, Numer. Algor. 74(4), 1169-1201 (2017)

[19] C. Chun and B. Neta, Appl. Math. Comput. 274, 762-773 (2016)

[20] J.R. Sharma and H. Arora, Appl. Math. Comput. 273, 924-933 (2016) 